\title{
Ethereum Blockchain Network Implementation for IoT Platform
}

\section{Mohamad Hafizat Zainal Abidin, Saravid Suchaad, Koichiro Mashiko*, Nordinah Ismail}

Electronic System Engineering, Malaysia-Japan International Institute of Technology,

UTM Kuala Lumpur, Jalan Sultan Yahya Petra, Kampung Datuk Keramat, Kuala Lumpur, 54100, MALAYSIA

*Corresponding Author

DOI: https://doi.org/10.30880/ijie.2019.11.07.001

Received 30 May 2019; Accepted 30 July 2019; Available online 10 August 2019

\begin{abstract}
Internet of things (IoT) is becoming increasingly important and ubiquitous in home and industries. It makes object a part of the Internet and fuses the digital and physical world together. However, if not properly secured, IoT device can be vulnerable to tampering that can change the content of the data stored in the network. Blockchain, a technology used in cryptocurrency, can be used for addressing the security and privacy challenges in IoT. Blockchain can be beneficial to IoT by adding more security layer and reducing dependency on a central authority. This paper proposed to develop a blockchain based platform for IoT application. Blockchain technology can be utilized to provide a decentralized network, creating a more secured IoT system. The proposed blockchain network will be based on Ethereum blockchain network allowing the use smart contract application for management of IoT devices. Experiment is conducted to show the feasibility of the blockchain based platform. The proposed blockchain based platform is capable of managing several IoT devices connected to the network.
\end{abstract}

Keywords: Blockchain, Internet of Things, security

\section{Introduction}

With the advancement in wireless and cloud technology, Internet of Things (IoT) is becoming more accessible in our daily life. These smart devices are interconnected together in a network which enables them to communicate and exchange data with each other [1]. Smart devices are capable of performing their function without the need of human intervention. Currently, there are various devices that are already in service such as medical monitoring equipment in hospitals, weather sensors, sensors in automobiles, smart homes, surveillance system and animal identification implant biochips that are already serving society needs [2,3]. This shows the significance of IoT in society due to its diverse application in our everyday life. It is foreseen that IoT technology will be applied to more and more devices to create a global network of Internet-connected devices.

Currently, most IoT system is based on a centralized system where data from sensor and actuator are analysis and monitor in a central control unit. However, IoT data that travel through multiple nodes in a centralize network are vulnerable to attack that compromises nodes in the network. To ensure the constant availability of IoT network, it is necessary to develop a tamper-proof environment for IoT to prevent attacks on IoT devices that may hinder the availability of the network. A continuously growing IoT networks may also be vulnerable to single points of failure which may in turn deteriorate the services provided by the IoT. Implementing a fault-tolerant mechanism for a large number of IoT devices is required in the network to prevent failure [4]. Moreover, some IoT scenarios are unsuitable for a centralize system due the IoT devices being mobile and constantly changing their network during their lifetime [5]. This can lead to some security issue for the IoT devices. Information in a centralize network can be tampered and forged by compromising the nodes which can leads to distrust [6]. This is even more crucial when the IoT system is responsible for 
monitoring various systems. A solution to improve the security of IoT is to implement blockchain technology into IoT application.

In this paper, we propose a decentralized blockchain based platform for IoT application. The proposed blockchain based platform uses a control unit that will manage IoT device and maintain the blockchain network by constantly validating transaction. With the use of block chain technology, the proposed platform will enables IoT devices to interact with each other in a decentralized and trustless network without the need for a third party intermediary.

In the following Section 2, we will discuss about the background of blockchain. Section 3 describes the methodology for the proposed blockchain based platform. Section 4 show the result and discussion from the proof of concept experiment of the proposed blockchain based platform and Section 5 conclude the article.

\section{Blockchain}

Blockchain technology has been foreseen to revolutionize IoT industry in managing, controlling, and most importantly securing IoT devices. This section gives a brief background about blockchain, and describes how blockchain can be a key enabling technology for providing viable security solutions to IoT security issue.

\subsection{Background}

Blockchain is a decentralized distributed ledger where transactions are recorded and sequentially grouped into blocks to be distributed to all nodes participating in the system [7]. It was first implemented by Satoshi Nakamoto in the cryptocurrency Bitcoin as a solution to the double-spending problem. Blockchain is a completely transparent technology that can show all the transactions that have been made since its creation, without tampering or fraud. The transaction in a blockchain network is validated and maintained by a network of participants called miners according to a predefined consensus mechanism (a set of rules allowing the network to reach a global agreement) [8]. The miners are actively involved in verifying and validating transactions in the network. This allows a blockchain network to be decentralized without the need of a central authority and eliminates the need for third party verification [9]. Once a transaction is validated and verified by majority consensus, the data block is inserted into the blockchain.
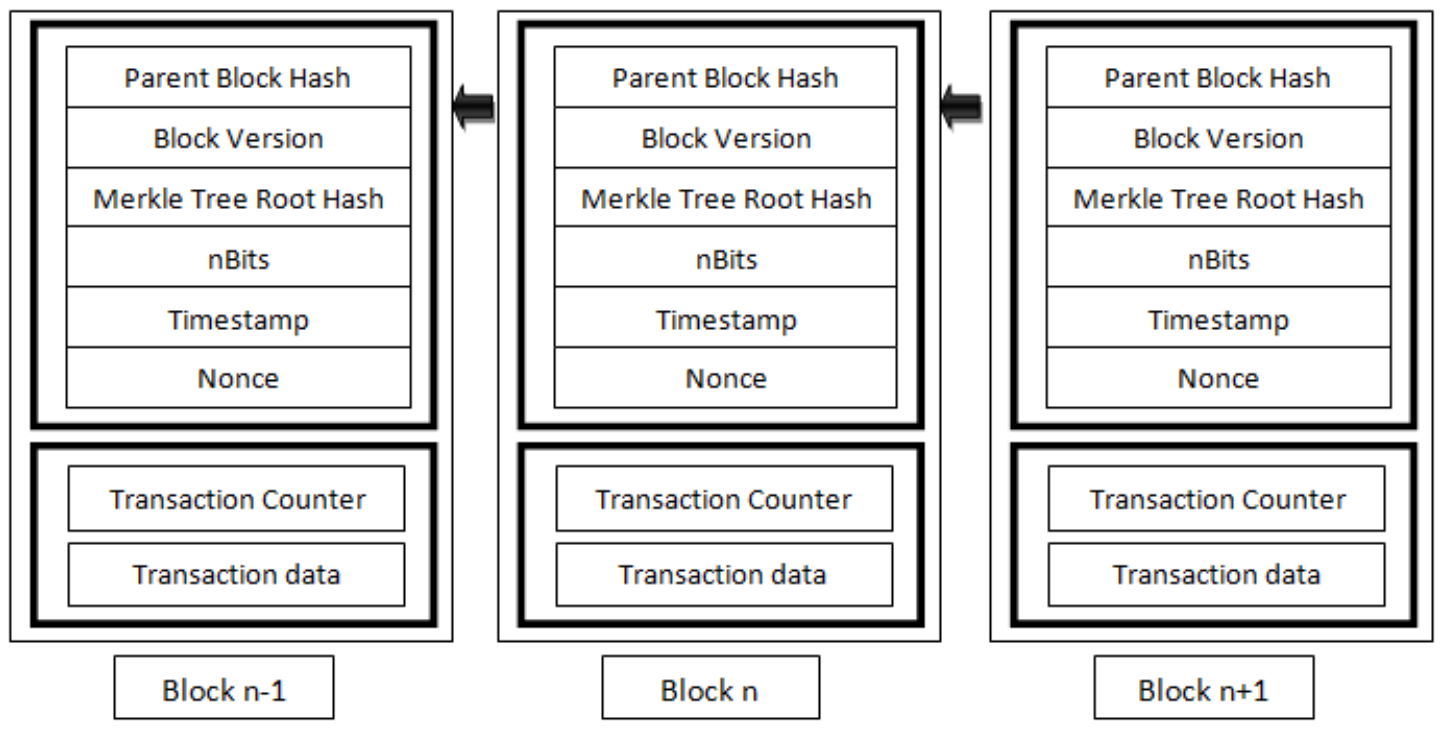

Fig. 1 - A blockchain structure.

Blockchain consist of a sequence of blocks that contain the record of all transaction [10]. Each block is chained to the previous one and recorded across a peer-to-peer network, using cryptographic trust and assurance mechanisms. A typical blockchain block consists of the block header and the block body. Fig. 1 illustrates an example of a blockchain design structure showing chained blocks. The block header includes the parent block hash, block version, Merkle tree root hash (the hash value of all the transactions in the block), nBits (target threshold of a valid block hash), time stamp and nonce (a 4-byte field) [10]. The block body consists of a transaction counter and the transactions [10]. The maximum number of transactions that a block can contain depends on the block size and the size of each transaction.

Blockchain technologies have the following advantages compare to centralize systems:

- Decentralized: Compared with the centralized network structure, every node in a blockchain network has a copy of the blockchain and cooperates with each other to maintain the blockchain. This allows for blockchain system 
to operate as a decentralized system without the need for a central authority or third-party intermediary. Single point of failure can be effectively mitigated by using blockchain.

- Tamper proof: Since each node in the network has a copy of the blockchain and the chain structure properties of the blockchain, malicious users attempting to modify one block stored in the blockchain, have to rebuild the whole chain after it, which exponentially increases the time taken to tamper it.

- Transparent and auditability: The blockchain record and store the full copy of every transaction ever executed in the network. All the data stored in the blockchain is publicly accessible to all the nodes in the network.

Blockchain has the potential to revolutionize the IoT technology with various implementation and applications for IoT. Some of these applications of blockchain technology for IoT include automobile, surveillance system, healthcare management, energy management, manufacturing automation, agriculture automation, transportation and logistics, finance security, sharing economy and smart city [11].

\section{Methodology}

The aim of this research is to design and deploy a blockchain based platform for IoT. The blockchain network will be connected to numerous numbers of IoT systems that are communicating and exchanging data with each other. The proposed blockchain based platform can be divided into four different components:

- IoT Devices: IoT devices includes various small wireless devices that can either contain built in sensor, actuator or motor. IoT devices function to record information from sensor and transferring the information to the control unit. The devices are not involved in the mining process of the blockchain. This is due to their limited computational power, memory storage and battery.

- Blockchain Network: The blockchain network in the blockchain based platform is a private blockchain for the sake of simplicity. This will provide better and more reliable result when evaluating the system. Control unit in the network act as miner to keep the network stable and secure by approving transaction and updating the blockchain ledger. However, in a real IoT scenario, a public blockchain should be used instead for the proposed platform.

- Smart Contract: The management of the blockchain network is control by the function defined in the smart contract. The smart contract is deployed in the blockchain network and is triggered when a transaction (data exchange) for the requirement in the contract is met. Once the function is triggered, the miners will keep the information of the transaction publicly accessible.

- Control Unit: The control unit function to control and monitor the IoT devices in the blockchain network. Beside, the control unit act as a miner in the network and are involved in verifying and recording all the transaction in the network. This requires the control unit to be capable of managing multiple numbers of IoT devices and cannot be constrained in performance.

To show the feasibility of the proposed platform, experiment will be conducted using computer (control unit) and Raspberry Pi (IoT devices) to simulate an IoT network. The experiment is conducted using a few IoT devices instead of hundreds of devices to show the proof of concept of the blockchain based platform.

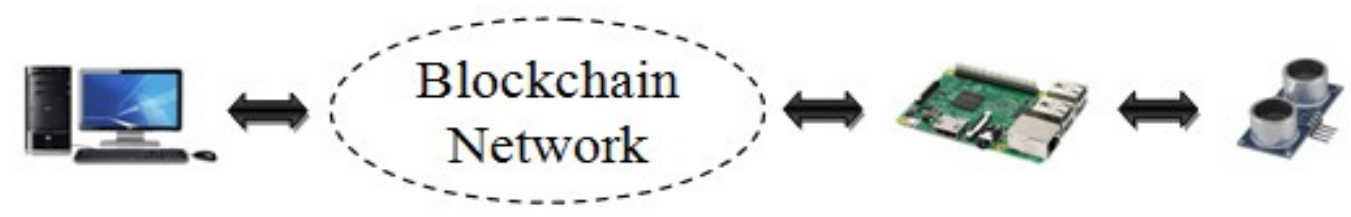

Fig. 2 - Experiment setup. Devices are connected to a router in a local connection

For the experiment, Ethereum is selected because it is an open and flexible blockchain that allow the development of decentralized IoT application. The computer and Raspberry Pi will also act as a member of the blockchain network and will synchronize the data in the blockchain ledger. Fig. 2 show the experiment setup for the experiment. Raspberry Pi will be connected to an ultrasonic sensor to simulate an IoT device that constantly measure and record sensory information. For the experiment, the Raspberry Pi will be exchanging sensory data with the computer through the blockchain network as shown in Figure 3. Go Ethereum (Geth) is installed into the computer and Raspberry Pi to allow the creation of the private blockchain network and smart contract for the experiment. Node.js and Web3 package is installed inside the Raspberry Pi to allow Raspberry Pi to interact with the smart contract in the blockchain. Node.js is used to execute the script that will interact with the smart contract. The Web3 package is a node module that provides a JavaScript API to interact with the blockchain and allow the JS script to fetch the status of the smart contract. The transaction between the computer and Raspberry Pi will be recorded in the blockchain. 
The experiment will be conducted using computer and Raspberry Pi with ultrasonic sensor connected through the blockchain network. Smart contract will be deployed in the network to manage the exchange between Raspberry Pi and computer. The smart contract will function to turn on the ultrasonic sensor and start measuring distance when input is send from the computer to the Raspberry Pi. The sensory data is then relay to the computer through the network.

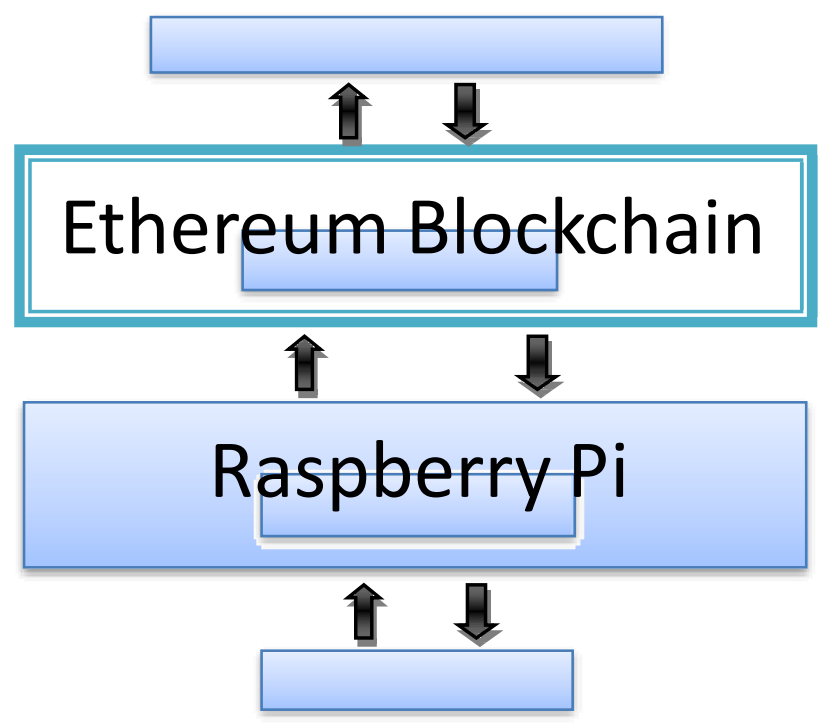

Figure 3: Data flow of the setup

\section{Result and Discussion}

The experiment was conducted with smart contract deployed in the network as shown in Figure 4. Once, the smart contract is deployed, input value is updated to start the Ethereum network. Data collection for the experiment can begin. The result from the proof of concept experiment shows the feasibility of the proposed blockchain based platform. The computer and Raspberry Pi in the blockchain network are able to communicate and exchange data with each other. Each device acts as a member of the blockchain member synchronizing with ledger in the network. The computer maintains the blockchain network by constantly validating transaction through the mining process. A sample of sensory data from the ultrasonic sensor that is retrieved from the Rasberry Pi is shown in Figure 5. From the experiment, blockchain can be used to create a secured wireless communication between IoT devices.

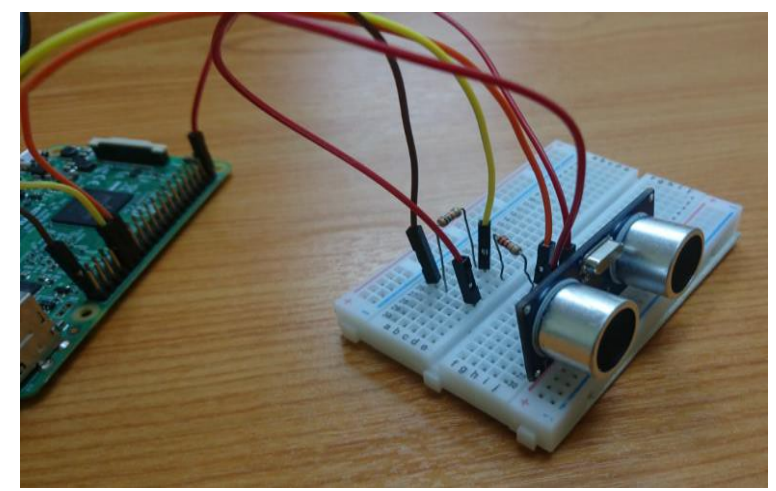

Figure 4: Raspberry Pi with sensor that will relay information to the computer 


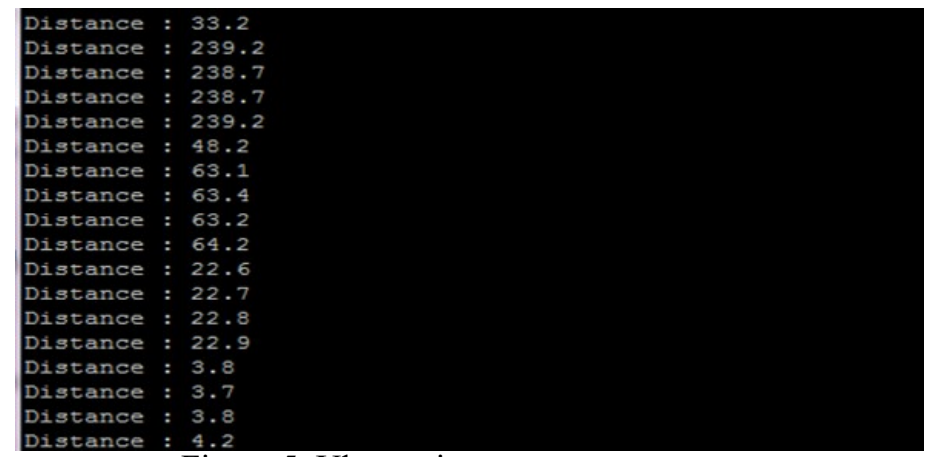

Figure 5: Ultrasonic sensor measurement

During development, some issues were discovered on the Ethereum blockchain. First, even though the transaction time process for the blockchain is 12 second, it is still sufficiently fast enough for some IoT scenarios. For IoT scenarios requiring instantaneous processing, implementing Ethereum might not be suitable and a different blockchain with faster transaction time. Second, implementing the entire public Ethereum blockchain might not be feasible unless IoT devices have larger memory storage. Although a private blockchain might be feasible, for the public blockchain, a lighter blockchain client is required where IoT devices do not need to store the entire blockchain.

\section{Conclusion}

In this paper, we propose a decentralized and scalable blockchain based platform for IoT application The proposed platform will enables IoT devices to interact with each other in a decentralized and trustless network without the need for a third party intermediary. IoT device will be able exchange data through a secure network, safe from possible attacks that might tamper with the device. The proposed blockchain-based platform uses a control unit to manage the various IoT devices connected in the network. The security of the network is ensured by the strong security properties of the blockchain technology. The proof of concept experiment conducted using computer and Raspberry Pi has shown the feasibility of the blockchain based platform. This paper presents a preliminary study on the blockchain technology and its potential applications in IoT. The work lays a foundation for our future work for more scalable and feasible blockchainbased platform for various IoT applications.

\section{Acknowledgement}

First and foremost, sincere thanks to Prof. Dr. Koichiro Mashiko and Puan Nordinah Binti Ismail, who have given invaluable knowledge, guidance, advice and time from the early stage until the final stage of this research. Moreover, a great gratitude goes to every individual that are involved, for your kindness, motivation, and cooperation in this research. This study is supported by Universiti Teknologi Malaysia.

\section{References}

[1] Md Yunus, M. A. (2017). Internet of Things (IoT) Application in Meliponiculture. International Journal of Integrated Enginering, 9(4).

[2] Gismalla, M. S. M., \& Abdullah, M. F. L. (2017). Device to Device Communication for Internet of Things Ecosystem: An overview. International Journal of Integrated Engineering, 9(4).

[3] Zhang, Y., \& Wen, J. (2016). The IoT electric business model: Using blockchain technology for the internet of things. Peer-to-Peer Networking and Applications, 10(4), 983-994.

[4] Ammar, M., Russello, G., \& Crispo, B. (2018). Internet of Things: A survey on the security of IoT frameworks. Journal of Information Security and Applications, 38, 8-27.

[5] Zhang, Y., \& Wen, J. (2016). The IoT electric business model: Using blockchain technology for the internet of things. Peer-to-Peer Networking and Applications, 10(4), 983-994.

[6] Conoscenti, M., Vetro, A., \& Martin, J. C. De. (2016). Blockchain for the Internet of Things : a Systematic Literature Review. 2016 IEEE/ACS 13th International Conference of Computer Systems and Applications (AICCSA), 1-6. [7]

Nguyen, Q. K. (2016). Blockchain - A Financial Technology for Future Sustainable Development. 2016 3rd International Conference on Green Technology and Sustainable Development (GTSD), 24-25 November. Kaohsiung, Taiwan, 51-54.

[8] Christidis, K., \& Devetsikiotis, M. (2016). Blockchains and Smart Contracts for the Internet of Things. IEEE Access, 4, 2292-2303.

[9] Novo, O. (2018). Blockchain Meets IoT: An Architecture for Scalable Access Management in IoT. IEEE Internet of Things Journal, 5(2), 1184-1195. 
[10] Zheng, Z., Xie, S., Dai, H., Chen, X, \& Wang, H. (2017). An Overview of Blockchain Technology: Architecture, Consensus, and Future Trends. 2017 IEEE International Congress on Big Data (BigData Congress), 25-30 June. Honolulu, HI, USA, 557-564.

[II] Kumar, N. M., \& Mallick, P. K. (2018). Blockchain technology for security issues and challenges in loT. Procedia Computer Science, 132, 1815-1823. 